\title{
Analysis of Disturbing Influence of Traffic Load on Soil Body
}

\author{
Janat Musayev ${ }^{1}$ and Algazy Zhauyt ${ }^{2}$ \\ ${ }^{1}$ Department of Transport Engineering and Technologies, Kazakh Academy of Transport and Communications Named after \\ M.Tynyshpayev, Almaty 050012, Kazakhstan \\ ${ }^{2}$ Department of Applied Mechanics and Basics of Machine Design, Kazakh National Technical University Named after K.I.Satpaev, \\ Almaty 050013, Kazakhstan \\ Correspondence should be addressed to Janat Musayev; mussaev1975@mail.ru
}

Received 18 October 2014; Revised 30 November 2014; Accepted 21 December 2014

Academic Editor: Binbin Weng

Copyright ( $) 2015$ J. Musayev and A. Zhauyt. This is an open access article distributed under the Creative Commons Attribution License, which permits unrestricted use, distribution, and reproduction in any medium, provided the original work is properly cited.

\begin{abstract}
Stress waves propagate in soil in case of earthquake and man-made effects (traffic flow, buried explosions, shield-driven pipes and tunnels, etc.). The wave point-sources are those located at the distances equal to more than two waves lengths, which significantly simplifies solving of a problem of these waves' strength evaluation. Distribution of stress and displacement by the stress waves propagation in elastic medium is a complex pattern. The stress distribution in propagating waves depends on the type and form of source, conditions of the source contact with medium, and properties of mediums in the vicinity of the source. The point-sources and their combinations are selected in such a way to model an influence of machines and processes on soil body in case of shielddriven pipes (tunnels).
\end{abstract}

\section{Introduction}

In confined environment the wave pattern becomes more difficult due to the boundary reflection of waves. For evaluation of different factors influence on the nature of waves propagation it is convenient to divide the waves propagation problem into several stages.

At the first stage tasks of waves propagation in infinite medium from the point-sources of the following different types are reviewed:

(i) concentrated force,

(ii) double force without moment (two concentrated forces acting contrariwise along one line and applied at small distance $2 \mathrm{~h}$ from each other),

(iii) two double forces without moment (double couple without moment) acting at right angles and in a single plane,

(iv) three double forces without moment acting in three orthogonally related directions (center of expansion),

(v) double forces with moment (couple of forces), (vi) combination of two couples of forces with sum of moments equal to zero,

(vii) uniform pressures in alveole at a section in length $d$,

(viii) tangential stresses applied at a section of alveole contour in length $d$,

(ix) tangential stresses applied to alveole contour in length $d$.

Solution of tasks enables evaluation of types of wave sources' influence on the type and parameters of waves propagating over a distance.

At the second stage tasks of wave propagation from the point-sources in semi-infinite elastic medium are reviewed. At this stage tasks of vibration of semi-infinite elastic medium surface from different point-sources of waves at various depths with account of waves reflected from free surface are solved. The solutions make it possible to evaluate a dynamic impact on the environment in case of earthquake, as well as the dynamic impact of different devices and machines used for underground works. This paper deals with a task of waves propagation from the point-sources of different types in the 
infinite space. In the performance of the tasks the Fourier integral transform and generalized functions are used [1-3].

\section{Materials and Methods}

For dynamic tasks both infinite and semi-infinite spaces and for bounded areas we will use differential equations of motion

$$
\mu U_{j, i i}+(\lambda+\mu) U_{i, i j}-\rho \ddot{U}_{j}=f_{j}, \quad i, j=1,2,3 .
$$

Suppose that in the generalized functions outside area $\Omega$ occupied by the elastic area the displacement and stress functions are equal to zero [1]. Then (1) may be in the form

$$
\begin{aligned}
\mu U_{j, i i} & +(\lambda+\mu) U_{i, i j}-\rho \ddot{U}_{j} \\
= & \mu\left\lfloor\left\lfloor U_{j}\right\rfloor \cos \left(n, x_{i}\right) \delta(s)\right\rfloor_{, i} \\
& +(\lambda+\mu)\left[\left[U_{i}\right] \cos \left(n, x_{i}\right) \delta(s)\right]_{j} \\
& +\left\lfloor\sigma_{j i}\right\rfloor_{s} \cos \left(n, x_{i}\right) \delta(s)-\rho\left[U_{k}\right]_{t=0} \\
& \times \dot{\delta}(t)+\rho\left[U_{j}\right]_{t=T} \dot{\delta}(t-T)-\rho\left[\dot{U}_{k}\right]_{t=0} \delta(t) \\
& +\rho\left[\dot{U}_{k}\right]_{t=T} \delta(t-T),
\end{aligned}
$$

where $\delta_{i j}$ is Kronecker symbol, $\delta_{s}$ is delta function at the area boundary, and $\delta(t), \dot{\delta}(t)$ are delta function and its time derivative. $\left\lfloor U_{j}\right\rfloor_{s}$ and $\left\lfloor\sigma_{i j}\right\rfloor_{s}$ are jump of $\left\lfloor U_{j}\right\rfloor$ and $\left\lfloor\sigma_{i j}\right\rfloor$ functions upon outside passage through the boundary of area $\Omega$. Since outside of this area these functions are equal to zero, symbols $\left\lfloor U_{j}\right\rfloor$ and $\left\lfloor\sigma_{i j}\right\rfloor$ present values of these functions at the area boundary.

Functions $\left[U_{j}\right]_{t=0},\left[U_{j}\right]_{t=T},\left[\dot{U}_{j}\right]_{t=0},\left[\dot{U}_{j}\right]_{t=T}$ represent initial and final conditions, that is, displacements and velocities of medium points at $t=0, t=T$.

In the expressions below and above the summation over repeated indices is performed.

Let us introduce the notations:

$$
\begin{aligned}
X_{j}= & \mu\left[\left[U_{j}\right] \cos \left(n, x_{i}\right) \delta(s)\right]_{, i} \\
& +(\lambda+\mu)\left[\left[U_{i}\right] \cos \left(n, x_{i}\right) \delta(s)\right]_{, j} \\
& +\left\lfloor\sigma_{j i}\right]_{s} \cos \left(n, x_{i}\right) \delta(s)-\rho\left[U_{k}\right]_{t=0} \\
& \times \dot{\delta}(t)+\rho\left[U_{j}\right]_{t=T} \dot{\delta}(t-T)-\rho\left[U_{k}\right]_{t=0} \\
& \times \delta(t)+\rho\left[\dot{U}_{k}\right]_{t=T} \delta(t-T)+F_{j} .
\end{aligned}
$$

Expression (3) contains information on mass load and impacts on the medium boundary and initial conditions. If the medium motion is considered at the finite interval $(0, T)$ of time, the final conditions of displacements and velocities functions of the medium points at time $T$ are included, which represent unknown quantities; in the used method the solutions fulfill a role of "integration constants." $F_{j}$ coincides with $f_{j}$ in area $\Omega$ and in interval $(0, T)$ and is equal to zero outside of this area and interval.
Since all functions represented in the finite functions are equal to zero outside the area, consequently jumps of functions at the area boundary are boundary conditions. Depending on the assigned tasks a part of these functions is set; the other is defined in the course of solution [4].

Let us apply the Fourier transform for the differential equation system solution. Let us multiply the left and right member of the equation by $\exp \left(i\left(\alpha_{k} x_{k}+\omega \tau\right)\right)$ and integrate with respect to four variables: $\left(x_{1}, x_{2}, x_{3}, \tau\right)$; in other words let us apply the Fourier transform to system of (2). Here $\alpha_{1}, \alpha_{2}, \alpha_{3}$ mean parameters of the Fourier transform spacewise, $\omega$ being frequency.

For description of the Fourier functions $\left(\sigma_{i j}, U_{j}, F_{j}\right)$ let in symbol $\left(\bar{\sigma}_{i j}, \bar{U}_{j}, \bar{F}_{j}\right)$. The following properties of the Fourier transform are used upon integration: $\partial U_{i} / \partial x_{j} \Rightarrow\left(-i \alpha_{j}\right) \widetilde{U}_{i}$ or in tonsorial notations $U_{i, j} \Rightarrow\left(-i \alpha_{j}\right) \widetilde{U}_{i}$ and $\ddot{U}_{i} \Rightarrow-\omega^{2} \widetilde{U}_{i}$.

Let us divide the left and right members of system of (2) by $\mu$ and introduce the notations: $\gamma^{2}=\alpha_{1}^{2}+\alpha_{2}^{2}+\alpha_{3}^{2}$ and $\beta=$ $\sqrt{(\lambda+2 \mu) / \mu}$ ratio of P-wave velocity $c_{1}=\sqrt{(\lambda+2 \mu) / \rho}$ to $\mathrm{S}$ wave velocity $c_{2}=\sqrt{\mu / \rho}$. Having used the agreed notations and the Fourier transform let us represent the system of (2) in the form

$$
\left(\gamma^{2}-\omega^{2} \beta^{2}\right) \widetilde{U}_{k}+\left(\beta^{2}-1\right) \alpha_{k} \alpha_{l} \widetilde{U}_{l}=\frac{\widetilde{X}_{k}}{\mu} .
$$

In the right member of (4) $\widetilde{X}_{k}$ includes not only the force impacts on medium but also kinematic ones.

Solving of algebraic equations system (4) may be written down in the following form:

$$
\widetilde{U}_{k}=\frac{\beta^{2}\left(\gamma^{2}-\omega^{2} \beta^{2}\right) \widetilde{X}_{j}-\left(\beta^{2}-1\right) \alpha_{j} \alpha_{k} \widetilde{X}_{k}}{\rho c_{1}^{2}\left(\gamma^{2}-\omega^{2} \beta^{2}\right)\left(\gamma^{2}-\omega^{2}\right)} .
$$

For detection of displacements it is necessary to perform an inverse Fourier transform:

$$
\begin{gathered}
U_{j}\left(x_{1}, x_{2}, x_{3}, \tau\right) \\
=\frac{1}{4 \pi^{2}} \cdot \int_{W} \frac{\beta^{2}\left(\gamma^{2}-\omega^{2} \beta^{2}\right) \widetilde{X}_{j}-\left(\beta^{2}-1\right) \alpha_{j} \alpha_{k} \widetilde{X}_{k}}{\rho c_{1}^{2}\left(\gamma^{2}-\omega^{2} \beta^{2}\right)\left(\gamma^{2}-\omega^{2}\right)} \\
\cdot e^{-\left(x_{k} \alpha_{k}+\omega \tau\right)} d W,
\end{gathered}
$$

where $W$ means a space of variables $\left(\alpha_{1}, \alpha_{2}, \alpha_{3}, \omega\right)$ and $d W=$ $d \alpha_{1}, d \alpha_{2}, d \alpha_{3}, d \omega$

For the infinite space symbols $X_{j}$ and $\widetilde{X}_{j}$ have only a generalized load of $F_{j}$ and $\widetilde{F}_{j}$.

\section{Results and Discussion}

Let us consider the method of solution of the tasks of waves propagation in the infinite elastic medium from the pointsources with application of the Fourier transform and generalized functions from the point-sources using the example of a task of the concentrated force $F(t)$ effect for comparison with famous Love solution [5]. 
Upon effect of the concentrated force at the origin of coordinates and in $x_{3}$-direction expressions (3) take the form

$$
X_{l}=0, \quad X_{2}=0, \quad X_{3}=F(t) \delta\left(x_{3}\right),
$$

and correspondingly the Fourier transform $\widetilde{X}_{3}=\widetilde{F}(\omega)$.

Under such conditions the displacement components take the form

$$
\begin{aligned}
& U_{1}\left(x_{1}, x_{2}, x_{3}, \tau\right) \\
& =-\frac{\beta^{2}-1}{4 \pi^{2} \rho c_{1}^{2}} \int_{W} \frac{\alpha_{1} \alpha_{3} \widetilde{X}_{3}}{\left(\gamma^{2}-\omega^{2} \beta^{2}\right)\left(\gamma^{2}-\omega^{2}\right)} \\
& \cdot e^{-\left(x_{k} \alpha_{k}+\omega \tau\right)} d W, \\
& \begin{aligned}
U_{2}\left(x_{1}, x_{2}, x_{3}, \tau\right) \\
=-\frac{\beta^{2}-1}{4 \pi^{2} \rho c_{1}^{2}} \int_{W} \frac{\alpha_{2} \alpha_{3} \widetilde{X}_{3}}{\left(\gamma^{2}-\omega^{2} \beta^{2}\right)\left(\gamma^{2}-\omega^{2}\right)} \\
U_{3}\left(x_{1}, x_{2}, x_{3}, \tau\right) \\
=\frac{1}{4 \pi^{2} \rho c_{1}^{2}} \cdot \int_{W} \widetilde{X}_{3} \frac{\beta^{-\left(x_{k} \alpha_{k}+\omega \tau\right)} d W,}{\left(\gamma^{2}-\omega^{2} \beta^{2}\right)-\left(\beta^{2}-1\right) \alpha_{3}^{2}} \\
\cdot e^{-\left(x_{k} \alpha_{k}+\omega \tau\right)} d W .
\end{aligned}
\end{aligned}
$$

Let us introduce the notation: $r=\sqrt{x_{1}^{2}+x_{2}^{2}+x_{3}^{2}}$.

Considering radiation conditions and using asymptotic development [2] of the Fourier integrals (8) and neglecting components representing fluctuation of near-field we will get

$$
\begin{aligned}
U_{1}= & \frac{1}{4 \pi \rho r} \frac{\partial^{2} r}{\partial x_{1} \partial x_{3}} \\
& \cdot\left[\frac{1}{c_{1}^{2}} F\left(t-\frac{r}{c_{1}}\right)-\frac{1}{c_{2}^{2}} F\left(t-\frac{r}{c_{2}}\right)\right], \\
U_{2}= & \frac{1}{4 \pi \rho r} \frac{\partial^{2} r}{\partial x_{2} \partial x_{3}} \\
& \cdot\left[\frac{1}{c_{1}^{2}} F\left(t-\frac{r}{c_{1}}\right)-\frac{1}{c_{2}^{2}} F\left(t-\frac{r}{c_{2}}\right)\right], \\
U_{3}= & \frac{1}{4 \pi \rho r} \frac{\partial^{2} r}{\partial x_{3}^{2}} \\
& \cdot\left[\frac{1}{c_{1}^{2}} F\left(t-\frac{r}{c_{1}}\right)-\frac{1}{c_{2}^{2}} F\left(t-\frac{r}{c_{2}}\right)\right] .
\end{aligned}
$$

We point out that it is possible to get more complete transforms of integrals (8). In such case there are solutions fully coinciding with Love solutions [5] for the concentrated force in the infinite space. This paper deals with propagation of stress waves from the point-sources of different types in the infinite space based on the asymptotic development of Fourier integrals.
In consequence of symmetry of displacements and stresses about axis $x_{3}$ it is possible to get rather convenient expressions if a spherical coordinate system going through the coordinates center is applied and $\varphi$ will be defined as an angle between the radial coordinate and positive axis $x_{3}$ :

$$
\begin{aligned}
& U_{r}=\frac{\cos \varphi}{4 \pi \rho r c_{1}^{2}} F\left(t-\frac{r}{c_{1}}\right), \\
& U_{\theta}=0, \\
& U_{\varphi}=\frac{\sin \varphi}{4 \pi \rho r c_{2}^{2}} F\left(t-\frac{r}{c_{2}}\right) .
\end{aligned}
$$

An interesting fact will be pointed out: over all distances from the point of force cross motions perpendicular to the force line $\varphi=\pi / 2$ exceed the longitudinal motion directed along the force line $(\varphi=0)$ :

$$
\frac{U_{\varphi}(r, \pi / 2)}{U_{r}(r, 0)}=\frac{c_{2}^{2}}{c_{1}^{2}} .
$$

The achieved method is used upon getting of functions describing wave propagation from different sources. Wave radiation patterns from such sources are provided below with the required clarifications. Propagation of stress waves from the point-sources of different types acting in the infinite elastic medium. Figures 1-10 have been obtained with use of MATLAB program complex [6-11].

3.1. Waves Propagation from Concentrated Force. Consider

$$
\begin{aligned}
& u_{r}=\frac{\cos \phi}{4 \pi \rho c_{1}^{2} r} F\left(t-\frac{r}{c_{1}}\right), \\
& u_{\theta}=0, \\
& u_{\phi}=\frac{\sin \phi}{4 \pi \rho c_{2}^{2} r} F\left(t-\frac{r}{c_{2}}\right) .
\end{aligned}
$$

3.2. Propagation of Waves from Combination of Two Forces (Double Force without Moment). Consider

$$
\begin{aligned}
& u_{r}=\frac{2 h \cos ^{2} \phi}{4 \pi \rho c_{1}^{3} r} F^{\prime}\left(t-\frac{r}{c_{1}}\right), \\
& u_{\theta}=0, \\
& u_{\phi}=\frac{2 h \sin \phi \cos \phi}{4 \pi \rho c_{2}^{3} r} F^{\prime}\left(t-\frac{r}{c_{2}}\right) .
\end{aligned}
$$

3.3. Propagation of Waves from Combination of Two Double Forces (Two Double Forces without Moment). Consider

$$
\begin{aligned}
& u_{r}=\frac{2 h \sin ^{2} \phi}{4 \pi \rho c_{1}^{3} r} F^{\prime}\left(t-\frac{r}{c_{1}}\right), \\
& u_{\theta}=0, \\
& u_{\phi}=\frac{2 h \sin \phi \cos \phi}{4 \pi \rho c_{2}^{3} r} F^{\prime}\left(t-\frac{r}{c_{2}}\right) .
\end{aligned}
$$




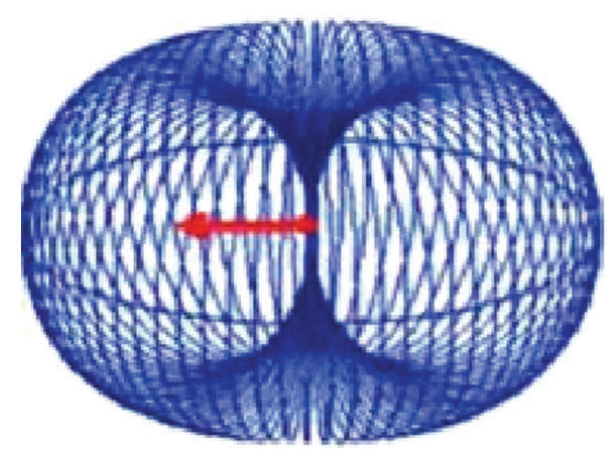

(a) P waves

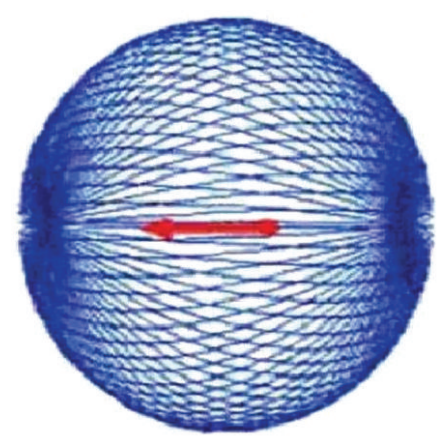

(b) $\mathrm{S}$ waves

FIGURE 1: Diagrams of waves propagation from concentrated force.

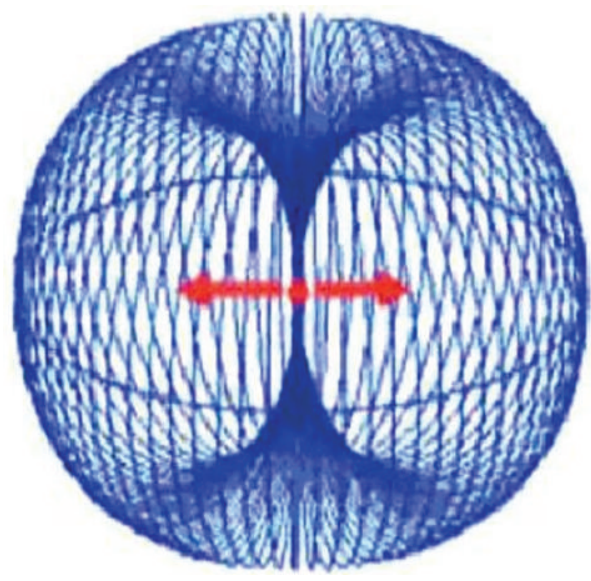

(a) P waves

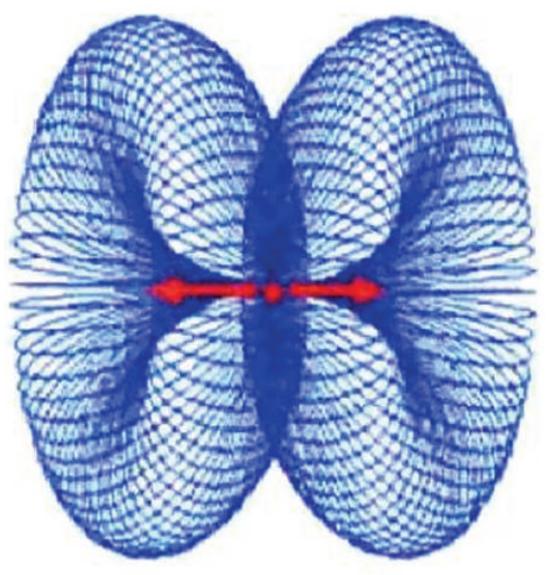

(b) $\mathrm{S}$ waves

FIGURE 2: Diagrams of waves propagation from combination of two forces.

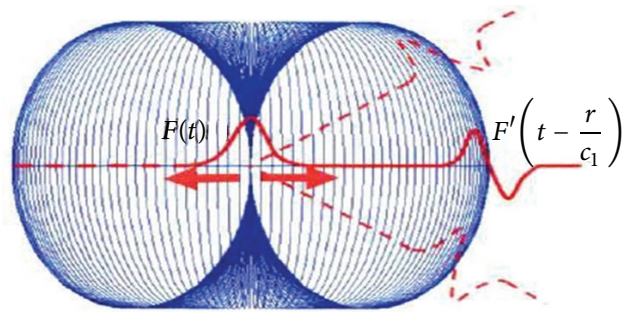

Figure 3: Clarification to diagram of waves propagation from combination of two forces without moment.

3.4. Propagation of Waves from Combination of Three Couples of Forces without Moment Directed in Parallel with Three Orthogonal Axes. Consider

$$
\begin{aligned}
& u_{r}=\frac{2 h}{4 \pi \rho c_{1}^{3} r} F^{\prime}\left(t-\frac{r}{c_{1}}\right), \\
& u_{\theta}=0, \\
& u_{\phi}=0 .
\end{aligned}
$$

3.5. Propagation of Waves from Two Couples of Forces. Consider

$$
\begin{aligned}
& u_{r}=0, \\
& u_{\theta}=-\frac{2 h \sin \phi}{4 \pi \rho c_{2}^{3} r} F^{\prime}\left(t-\frac{r}{c_{2}}\right), \\
& u_{\phi}=0 .
\end{aligned}
$$

3.6. Propagation of Waves from Combination of Two Couples of Forces with Sum of Moments Equal to Zero. Consider

$$
\begin{aligned}
& u_{r}=\frac{2 h \sin \theta \cos \theta \sin ^{2} \phi}{4 \pi \rho c_{1}^{3} r} F^{\prime}\left(t-\frac{r}{c_{1}}\right), \\
& u_{\theta}=\frac{2 h \sin \phi\left(\cos ^{2} \theta-\sin ^{2} \theta\right)}{4 \pi \rho c_{2}^{3} r} F^{\prime}\left(t-\frac{r}{c_{2}}\right), \\
& u_{\phi}=\frac{2 h \sin \theta \cos \theta \sin \phi \cos \phi}{4 \pi \rho c_{2}^{3} r} F^{\prime}\left(t-\frac{r}{c_{2}}\right) .
\end{aligned}
$$




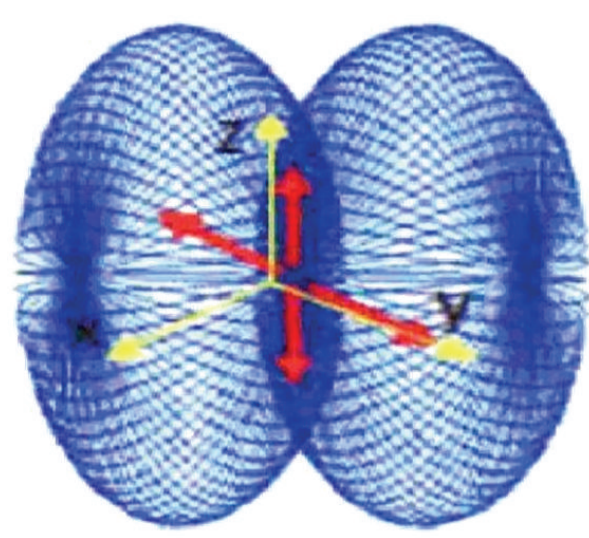

(a) P waves

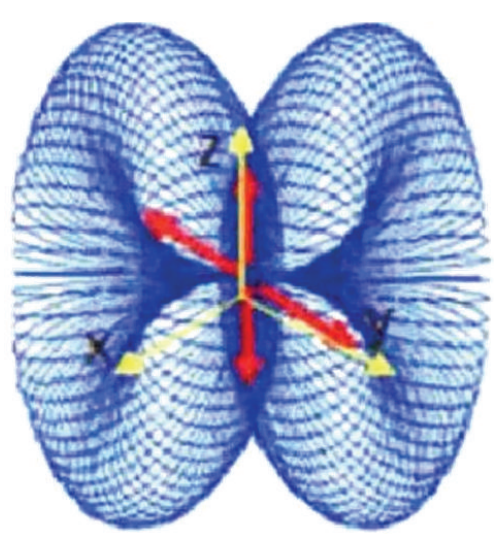

(b) S waves

FIGURE 4: Diagrams of waves propagation from combination of two double forces.
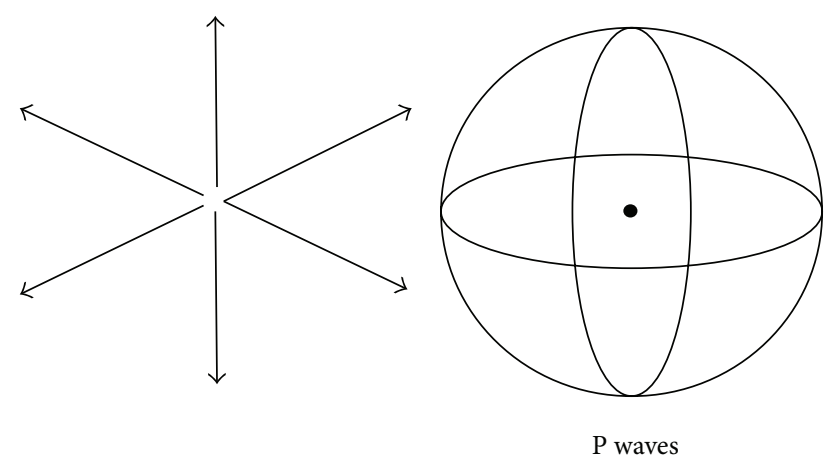

FIGURE 5: Diagrams of waves propagation from combination of three couples of forces.

3.7. Propagation of Waves Created by Uniform Pressure in Alveoleat a Sections in Length $d$. Consider

$$
\begin{aligned}
& u_{r}=\frac{\pi a^{2} d}{4 \pi \mu c_{1} r}\left(1-2 \frac{c_{2}^{2}}{c_{1}^{2}} \cos ^{2} \phi\right) p^{\prime}\left(t-\frac{r}{c_{1}}\right), \\
& u_{\theta}=0, \\
& u_{\phi}=\frac{\pi a^{2} d \theta \sin \phi \cos \phi}{2 \pi \mu c_{2} r} p^{\prime}\left(t-\frac{r}{c_{2}}\right) .
\end{aligned}
$$

3.8. Propagation of Waves Created by Tangential Stress at Alveole Contour in Length d. Consider

$$
\begin{aligned}
& u_{r}=0, \\
& u_{\theta}=\frac{\pi a^{2} d}{4 \pi \mu c_{2} r} \tau^{\prime}\left(t-\frac{r}{c_{1}}\right), \\
& u_{\phi}=0 .
\end{aligned}
$$

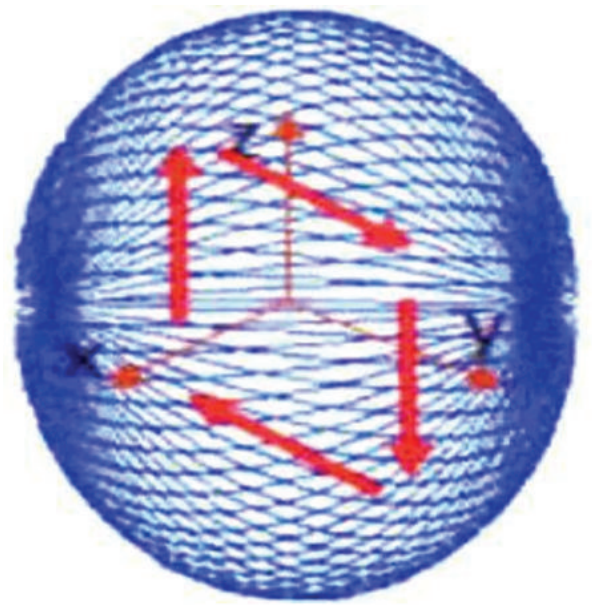

S waves

FIGURE 6: Diagrams of waves propagation from two couples of forces.

3.9. Propagation of Waves Created by Tangential Stress Applied to Alveole Contour at Section in Length d. Consider

$$
\begin{gathered}
u_{r}=\frac{2 \pi a d \cos \phi}{4 \pi \rho c_{1}^{2} r} p^{\prime}\left(t-\frac{r}{c_{1}}\right), \\
u_{\theta}=0, \\
u_{\theta}=0 \quad u_{\phi}=\frac{2 \pi a d \sin \phi}{4 \pi \rho c_{2}^{2} r} \tau\left(t-\frac{r}{c_{2}}\right) .
\end{gathered}
$$

Remark 1. Derivative with respect to all expressions above between the brackets is marked with a prime.

\section{Conclusions}

Solutions of stress waves propagation in elastic medium from different sources have been provided. 


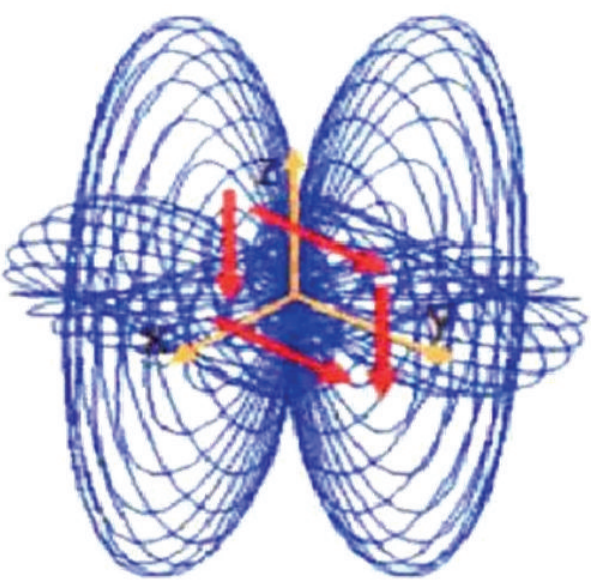

(a) P waves

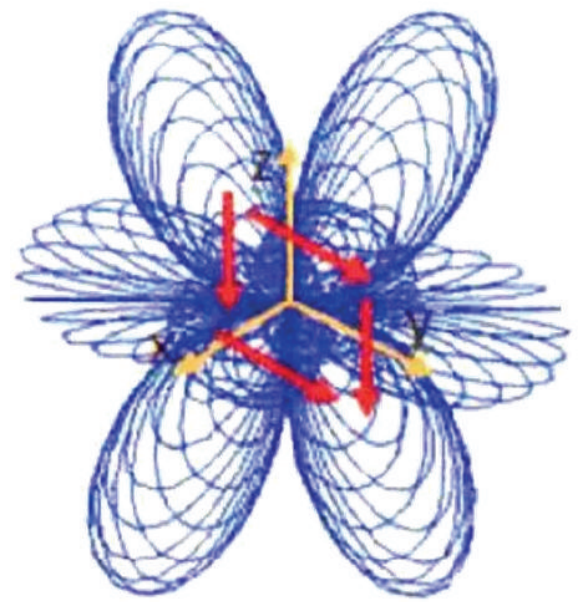

(b) S waves

Figure 7: Diagrams of waves propagation from combination of two couples of forces with sum of moments equal to zero.

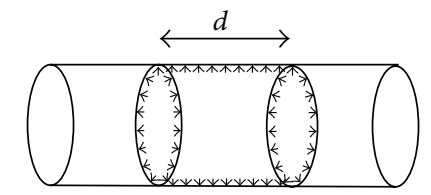

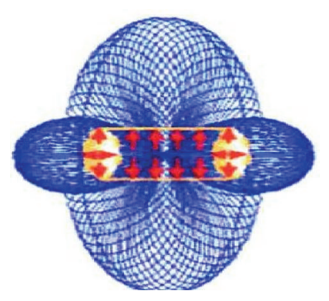

(a) P waves

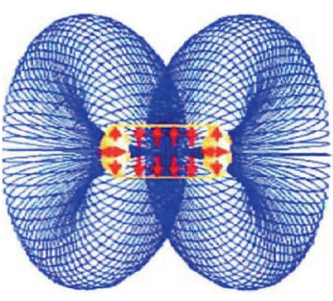

(b) S waves
FIGURE 8: Diagrams of propagation of waves created by uniform pressure.

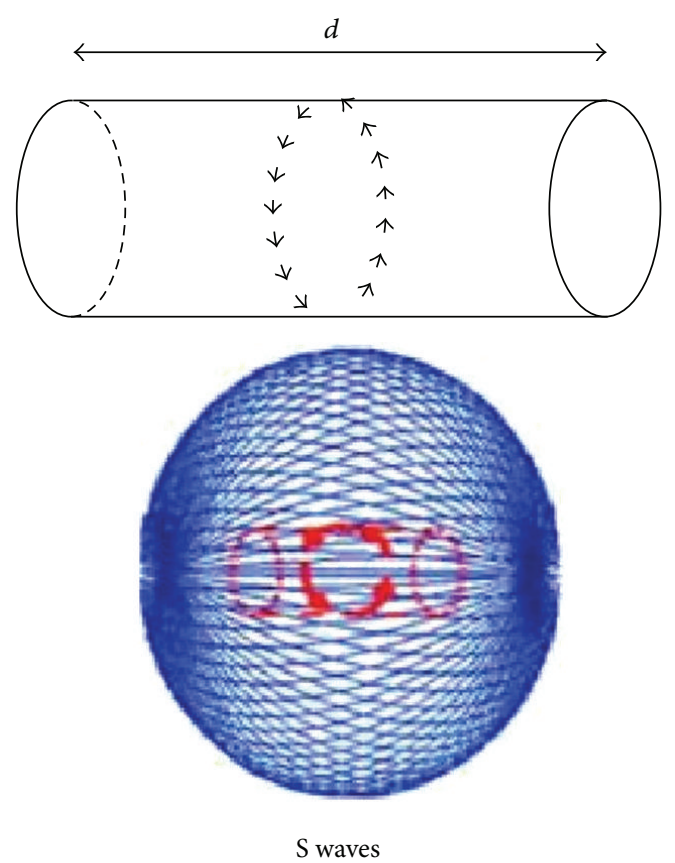

FIGURE 9: Diagrams of propagation of waves created by tangential stress at alveole contour in length $d$.

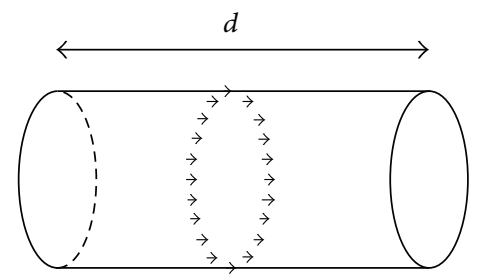

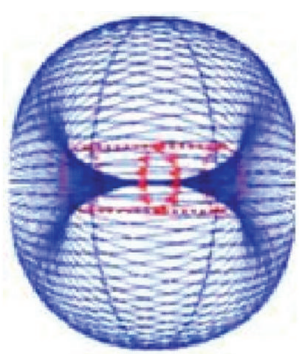

(a) P waves

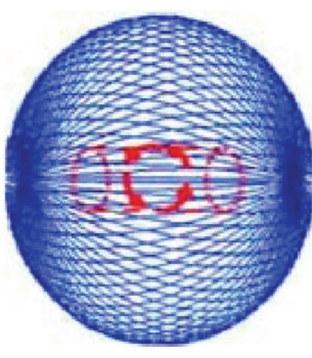

(b) S waves
FIGURE 10: Propagation of waves created by tangential stress applied to alveole contour.

The point-sources and their combinations have been selected in such a way to model the influence of machines and processes on soil body in case of shield-driven pipes (tunnels).

$\mathrm{P}$ waves and $\mathrm{S}$ waves propagate from each source of waves.

The waves propagate from concentrated force; the forms of these waves comply with the law of force variation. Wave amplitude decreases as $1 / r$ due to radiation in space.

Upon propagation of waves from double force without moment the wave forms represent a derivative of functions 
describing the law of force variation. The wave amplitude also decreases as $1 / r$ due to radiation in space.

The forms of waves propagating from different sources comply with the law of force variation and are derivatives of functions describing variation of forces and stresses.

\section{Conflict of Interests}

The authors declare that there is no conflict of interests regarding the publication of this paper.

\section{References}

[1] E. H. Kurbatskii, Method for solving problems of structural mechanics and the theory of elasticity, based on the properties of the Fourier image of finite functions [Ph.D. dissertation], MIIT, Moscow, Russia, 1995.

[2] A. Verruijt and J. R. Booker, "Surface settlements due to deformation of a tunnel in an elastic half plane," Geotechnique, vol. 46, no. 4, pp. 753-756, 1996.

[3] N. Loganathan and H. G. Poulos, "Analytical prediction for tunneling-induced ground movements in clays," Journal of Geotechnical and Geoenvironmental Engineering, vol. 124, no. 9, pp. 846-856, 1998.

[4] J. G. Wan g, S. L. Kong, and C. F. Leung, "Twin tunnels-induced ground settlement in soft soils," in Geotechnical Engineering in Urban Construction: Proceedings of the Sino-Japanese Symposium on Geotechnical Engineering, Beijing, China, 29-30 October 2003, pp. 241-244, Tsinghua University Press, 2003.

[5] A. D. Myshkis, "Mathematics for technical colleges," Special Courses, vol. 15, no. 6, pp. 632-644, 1996.

[6] K. M. Lee, R. K. Rowe, and K. Y. Lo, "Subsidence owing to tunnelling. I. Estimating the gap parameter," Canadian Geotechnical Journal, vol. 29, no. 6, pp. 929-940, 1992.

[7] K.-H. Park, "Analytical solution for tunnelling-induced ground movement in clays," Tunnelling and Underground Space Technology, vol. 20, no. 3, pp. 249-261, 2005.

[8] J. N. Franzius, Behavior of buildings due to tunnel induced subsidence [Ph.D. thesis], Department of Civil and Environmental Engineering, Imperial College of Science, Technology and Medicine, London, UK, 2003.

[9] O. B. John, T. R. Keusel, and E. H. King, Tunnel Engineering Handbook, Chapman \& Hall, 1996.

[10] Y. Leblais, Recommendations on Settlements Induced by Tunnelling, Association Française des Travaux en Souterrain (AFTES), 1995.

[11] T. W. Hulme, J. N. Shirlaw, and R. N. Hwang, "Settlements during the underground constructions of the Singapore MRT," in Proceedings of the 10th Southeast Asian Geo-Technical Conference, pp. 16-20, Taipei, Taiwan, 1990. 

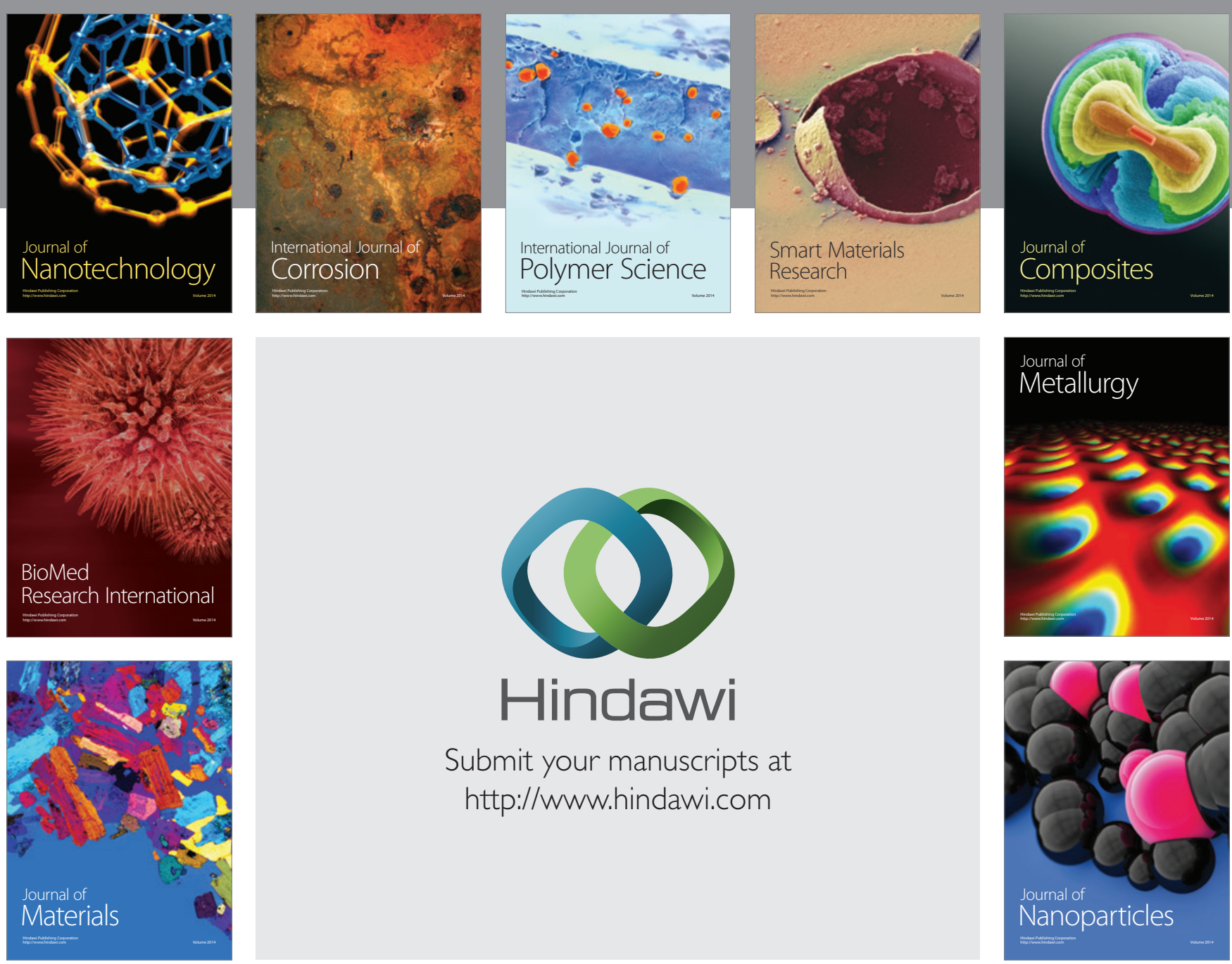

Submit your manuscripts at http://www.hindawi.com
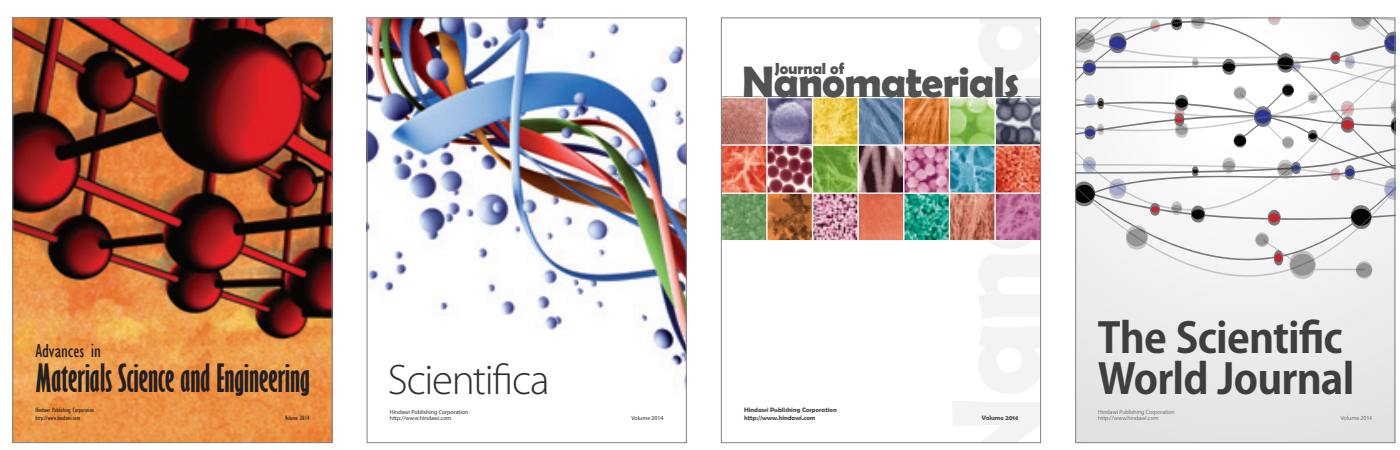

\section{The Scientific World Journal}
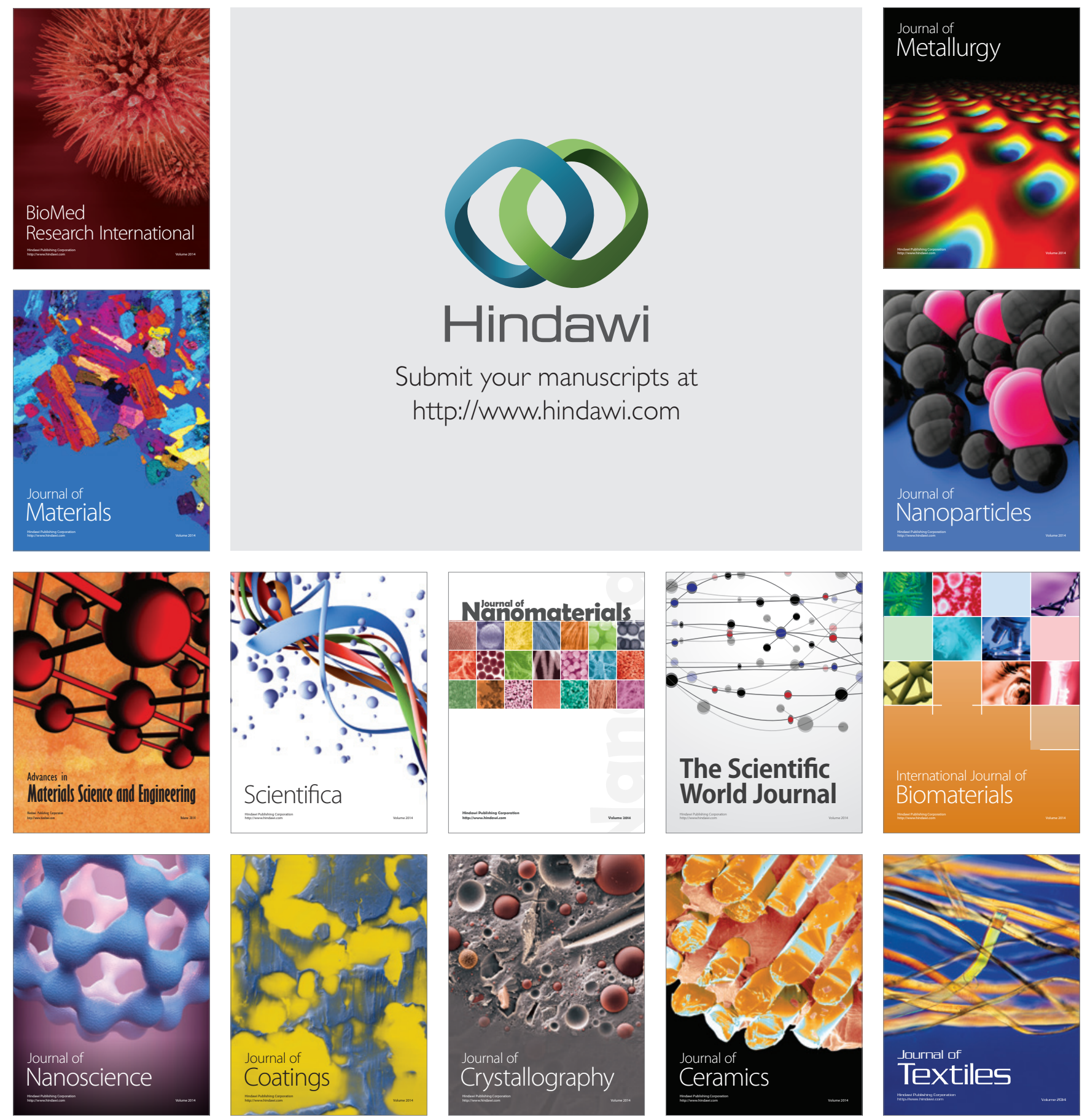